\title{
Implementing TOPSIS method in Selecting the Best Bus Transportation Services
}

\author{
Azmi bin Abu Seman ${ }^{1 *}$, Nasuha binti Alias ${ }^{2}$, Norfiza binti Ibrahim ${ }^{3}$ \\ ${ }^{1,2,3}$ Faculty of Computer and Mathematical Sciences \\ Universiti Teknologi MARA, Perlis Branch, Arau Campus, 02600 Arau, Perlis, Malaysia \\ Corresponding author: *azmi384@uitm.edu.my \\ Received Date: 1 October 2020 \\ Accepted Date: 12 October 2020
}

\begin{abstract}
Public transportation using express bus services are widely used by the public nowadays and many bus companies offer services to major cities as well as providing additional features and facilities to attract passengers. However, the passengers often face a problem in selecting the bus companies that give the best services for them according to their preferences. The purpose of this study is to develop a web application system that assists passengers in selecting the best bus services. The web application development follows the System Development Life Cycle (SDLC) methodology that contains four phases which are planning, designing, testing and implementing using PHP and MySQL database as tools for development. In order to get the best result, the application manipulates the MCDM approach using TOPSIS method. It enables users who already used the bus services to give the rating for each criterion and the users who want to use the bus services give their priority for each criterion. Based on TOPSIS rules, the web application will generate the result, which recommends the best bus transportation service for that particular user based on the input related to their preferences for each criterion. Usability testing is carried out in order to get feedback from the user, thus some refinements have been made to the interface of the system. User acceptance test was conducted after the refinement process. After the result was evaluated and analysed, it is shown that most of the components in the web application are well accepted by the respondents. The presence of this web application can help society to choose the best bus services that follow their preferences.
\end{abstract}

Keywords: bus transportation services, decision making, MCDM, TOPSIS method, web application

\section{INTRODUCTION}

Bus transportation services are widely used by people especially a person who likes to travel and also for students to go back to their school, college or university. However, they cannot choose the best bus transportation service based on their preferences because there is no guide to help them. Thus, this study developed a web based application system that can help passengers to choose the best bus transportation service based on criteria that they choose. The system can generate a result and display the bus companies that follows the criteria choosing by them.

In this study, Multi Criteria Decision Making (MCDM) approach is used to evaluate and choose the best bus transportation service. MCDM is a tool for evaluating problems and has the capability to review different alternatives such as choice, strategy, policy and scenario on numerous criteria for the best or the right alternative. MCDM approach has several methods such as Analytic Hierarchy Process (AHP) method, Elimination and Choice Translating Reality (ELECTRE) method, Technique for Order Preference by Similarly to Ideal Solution (TOPSIS) method, Weighted Sum Model (WSM) method, and Weighted Product Model (WPM) method. Each method is different and TOPSIS method was chosen in this study to select the service that follows the criteria based on passengers' preferences. 


\section{RESEARCH BACKGROUND}

\section{Multi Criteria Decision Making (MCDM)}

MCDM is an acronym for Multiple Criteria Decision Making. MCDM refers to an organized technique aimed at integrating multiple criteria into the steps include estimating, linking and grading dissimilar options (Li, 2013). According to Purjavad and Shirouyehzad (2011), MCDM is a process in making a decision with the existence of multiple purposes or goals. Moreover, the MCDM can be defined as related to structure, planning and solving a multiple criteria problem. The method will analyse a scope of dissimilar criteria, weight the criteria and choose the ideal one by using different methods of the MCDM (Aruldoss, Lakshmi, \& Venkatesan, 2013). Furthermore, the MCDM is a procedure to incorporate multiple indexes into one important index thus a ranking and the comparing are workable. A common problem that is ranked by the MCDM involves a number of alternatives that have to be evaluated and a number of criteria that have to evaluate the alternatives. The MCDM method has developed rapidly and is widely used in a research for complex decision problem that has to deal with multiple criteria (Lertprapai, 2013).

The TOPSIS method is selected for this study because it is easy to use compared to other methods and because this method has become popular. Other advantages apart from the ease of use are this method has a simple process and also is easy to programme. Other than that the TOPSIS method is easy to understand and has the capability to remain the same quantity of the steps although there is a problem about size (Velasquez \& Hester, 2013). Moreover, the TOPSIS method is used in application area that relates to supply chain management, engineering, manufacturing systems, business and marketing, environmental, human resources and water resources management (Velasquez \& Hester, 2013).

\section{Bus Transportation Services}

Commonly, passengers choose the bus services according to their preferences or criteria. According to Eboli and Mazzulla (2011) who developed the Customer Satisfaction Surveys (CSS), passengers are more comfortable to express their opinions about several attributes to describe the services through pre-defined measure of estimation. This judgement can also measure checking, estimating, and executing enhancements in transportation services.

\section{IMPLEMENTATION}

\section{TOPSIS Method}

A set of questionnaires was distributed among thirty (30) respondents in order to know what the most preferred criteria are by the users of bus transportation services. There are twenty (20) criteria listed in the questionnaire as shown in Table 1. The table indicates all the criteria and the number of selection of each criterion by the respondents. Finally, the top ten preferred criteria were chosen to be used in implementing TOPSIS method to develop the recommender web system.

Table 1: Preferred Criteria

\begin{tabular}{|l|l|c|}
\hline No & Criteria & Number of Selection \\
\hline $\mathbf{1}$ & Punctuality & 28 \\
\hline $\mathbf{2}$ & Ticket Price & 28 \\
\hline $\mathbf{3}$ & Cleanliness & 26 \\
\hline $\mathbf{4}$ & Driver Courtesy & 23 \\
\hline $\mathbf{5}$ & Appropriate Bus Schedule & 22 \\
\hline $\mathbf{6}$ & Wi-Fi Facilities & 22 \\
\hline $\mathbf{7}$ & Condition of Bus & 21 \\
\hline $\mathbf{8}$ & Comfort of Seat & 21 \\
\hline $\mathbf{9}$ & Safety of Luggage & 19 \\
\hline $\mathbf{1 0}$ & Seat Arrangement & 18 \\
\hline
\end{tabular}




\begin{tabular}{|l|l|c|}
\hline $\mathbf{1 1}$ & Online Ticketing & 14 \\
\hline $\mathbf{1 2}$ & Number of Stops Along Route & 13 \\
\hline $\mathbf{1 3}$ & Location of Rest Area & 10 \\
\hline $\mathbf{1 4}$ & Speed of Bus & 10 \\
\hline $\mathbf{1 5}$ & Drinks Provided & 6 \\
\hline $\mathbf{1 6}$ & Blanket Provided & 5 \\
\hline $\mathbf{1 7}$ & Entertainment & 5 \\
\hline $\mathbf{1 8}$ & Bus Suspension & 4 \\
\hline $\mathbf{1 9}$ & Quietness in Cabin & 4 \\
\hline $\mathbf{2 0}$ & Bus Body Manufacturer & 1 \\
\hline
\end{tabular}

Those criteria were implemented into the bus recommender system. There are several steps of the TOPSIS method has been used in order to calculate and choose the best bus services. According to Li (2013), the TOPSIS method is described as follows:

Step 1. Calculate the normalized decision matrix. The normalized value $n_{i j}$ is calculated as:

$$
n_{i j}=\frac{x_{i j}}{\sqrt{\sum_{i=1}^{m} x_{i j}^{2}}}, \quad i=1, \ldots, m, \quad j=1, \ldots, n .
$$

Step 2. Calculate the weighted normalized decision matrix.

The weighted normalized value $v_{i j}$ is calculated as:

$$
v_{i j}=w_{j} n_{i j}, \quad i=1, \ldots, m, \quad j=1, \ldots, n,
$$

where $w_{j}$ is the weight of the $i$ th criterion, and $\sum_{j=1}^{n} w_{j}=1$.

Step 3. Define the positive ideal solution (PIS) $A^{+}$and negative ideal solution (NIS) $A^{-}$as:

$$
A^{+}=\left\{v_{1}^{+}, \ldots, v_{n}^{+}\right\} \quad A^{-}=\left\{v_{1}^{-}, \ldots, v_{n}^{-}\right\}
$$

where, for benefit criterion:

$$
\begin{aligned}
& v_{j}^{+}=\max _{i}\left\{v_{i j}\right\}, \quad j=1,2, \ldots, n \\
& v_{j}^{-}=\min _{i}\left\{v_{i j}\right\}, \quad j=1,2, \ldots, n
\end{aligned}
$$

for cost criterion:

$$
\begin{gathered}
v_{j}^{-}=\max _{i}\left\{v_{i j}\right\}, \quad j=1,2, \ldots, n \\
v_{j}^{+}=\min _{i}\left\{v_{i j}\right\}, \quad j=1,2, \ldots, n
\end{gathered}
$$

Step 4. Calculate the distances $\mathrm{d}^{+}$and $\mathrm{d}^{-}$of each alternative from PIS and NIS using the following equations, respectively: 


$$
\begin{aligned}
& d_{i}^{+}=\sum_{j=1}^{n} \operatorname{dis}\left(v_{i j}-v_{j}^{+}\right), \quad i=1, \ldots, m \\
& d_{i}^{-}=\sum_{j=1}^{n} \operatorname{dis}\left(v_{i j}-v_{j}^{-}\right), \quad i=1, \ldots, m
\end{aligned}
$$

where, $\operatorname{dis}\left(v_{i j}-v_{j}^{+}\right)$is the distance between evaluation value of alternative $i$ and FPIS on the $j$ criterion, dis $\left(v_{i j}-v_{j}^{-}\right)$is the distance between evaluation value of alternative $i$ and NPIS on the $j$ criterion.

Step 5. Calculate the relative closeness to the ideal solution. The relative closeness $R_{i}$ of the alternative Ai with respect to $\mathrm{Ap}$ is defined as:

$$
R_{i}=\frac{d_{i}^{-}}{\left(d_{i}^{+}+d_{i}^{-}\right)}, \quad i=1, \ldots, m
$$

According to the relative closeness degree $R_{i}$, the ranking order of the alternatives can be determined. If any alternative has the highest $R_{i}$ value, then, it is the most desirable alternative.

\section{Formula Implementation}

A web based application system is developed to implement the TOPSIS method based on the ten (10) preferred criteria. The system enable users to give reviews about the bus transportation companies that they experienced before. In this study, four bus companies plying between Kangar to Kota Bharu route were selected. Those companies are Ekspres Mutiara, Ekspes Perdana, Transnasional and Pancaran Matahari. The researcher has approached ten passengers as respondents that have experienced the bus services with those companies to answer the criteria review through the system.

TOPSIS method requires the respondents to go to the 'Criteria Review' page, choose bus company and give a rating (tick) for each of the criteria either 'Very Poor', 'Poor', 'Medium', 'Good' or 'Very Good' as shown in Figure 1. The rating given by each passengers are stored in database.

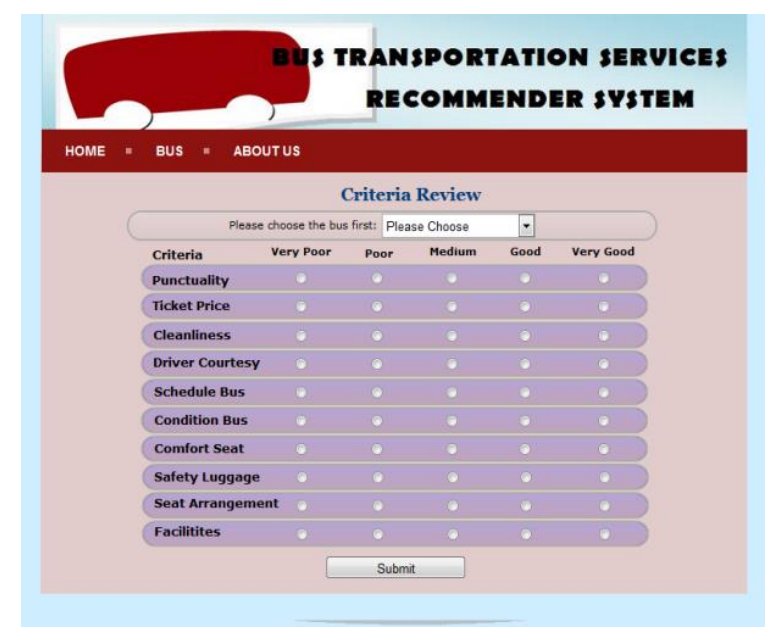

Figure 1: Criteria Review

The respondents (Passengers) who wish to choose the best bus services for their next trip can go to the 'Bus Recommender' page and choose the importances of each criteria, either 'Not Very Important', 'Not Important', 'Medium', 'Important' and 'Very Important', as shown in Figure 2. 


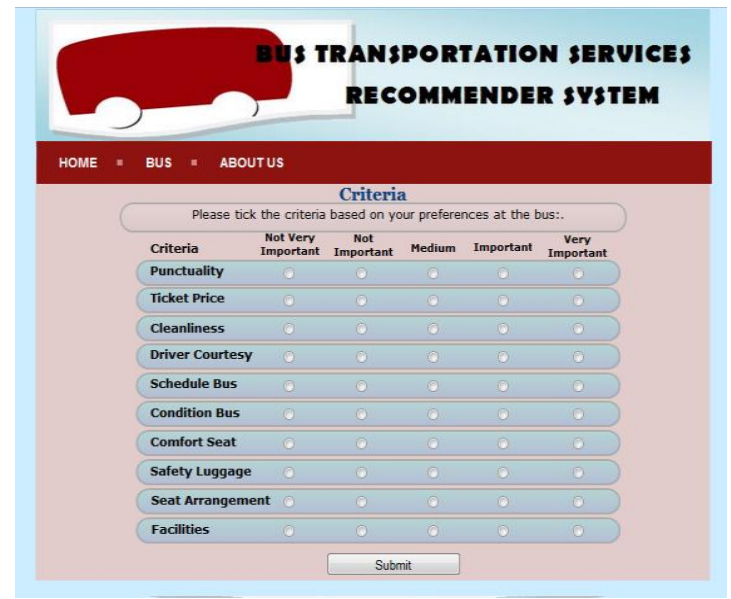

Figure 2: Importances of each Criteria

After that, a result is displayed where the best bus transportation service recommended by the system (calculated by using TOPSIS method) is shown as in Figure 3.

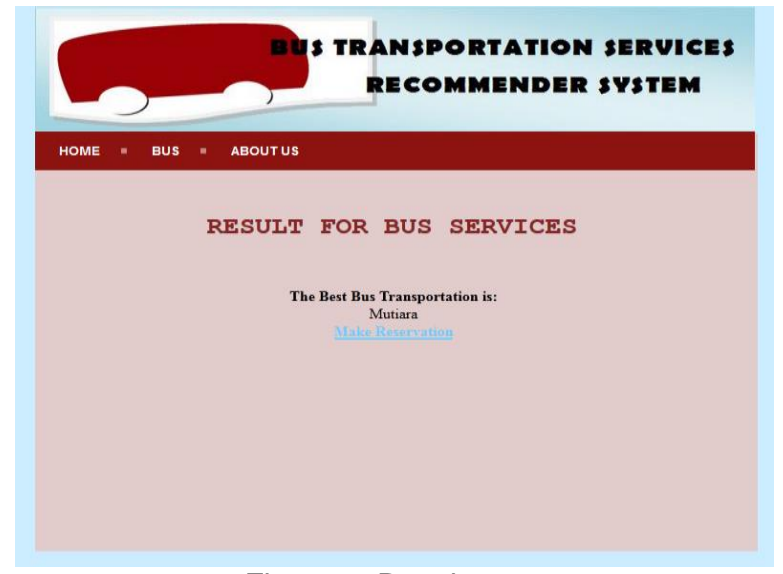

Figure 3: Result page

\section{RESULTS AND FINDINGS}

Usability testing is carried out in order to get feedback from the user when using the system. In order to conduct the usability testing, ten respondents were chosen from the IPT students that have experienced using the bus transportation services. The respondents have various family and social background. Based on observations and feedbacks of the respondents from usability testing, some of the improvement has been made to the interface of the system. Among the refinements are changing the visibility of the banner, simplified the instructions, organized the result properly, and put the details of bus in the table as shown in Figure 4. 


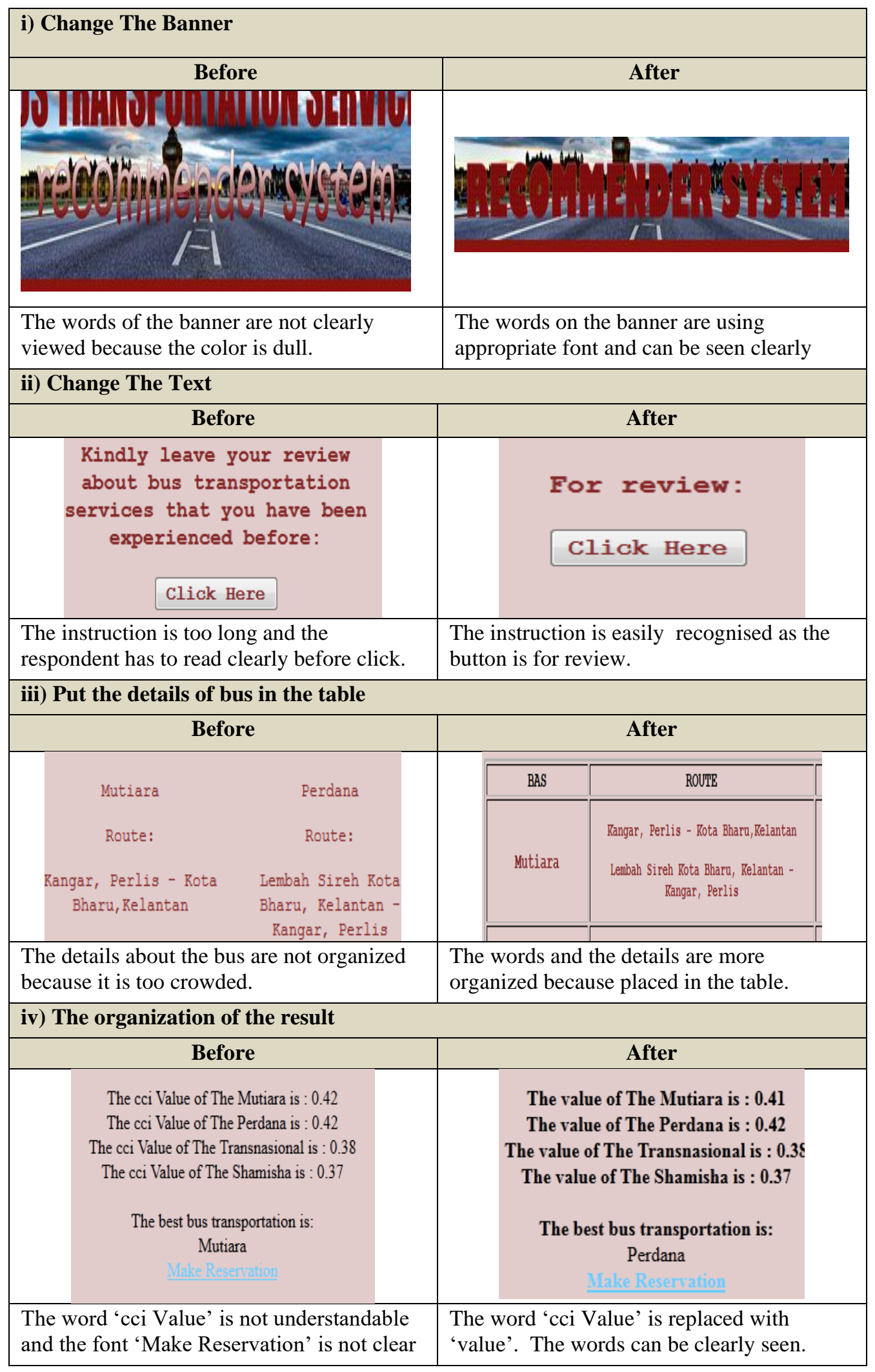

Figure 4: Refinement 
Next, user acceptance test was conducted after the refinement process in order to know the user's view in dealing with the web application. During the user acceptance test, the user was given the questionnaire and they have to answer it based on their perception about the web application system. The user from different background were involved in the testing. The result from user acceptance test was evaluated and analysed as shown in Figure 5.

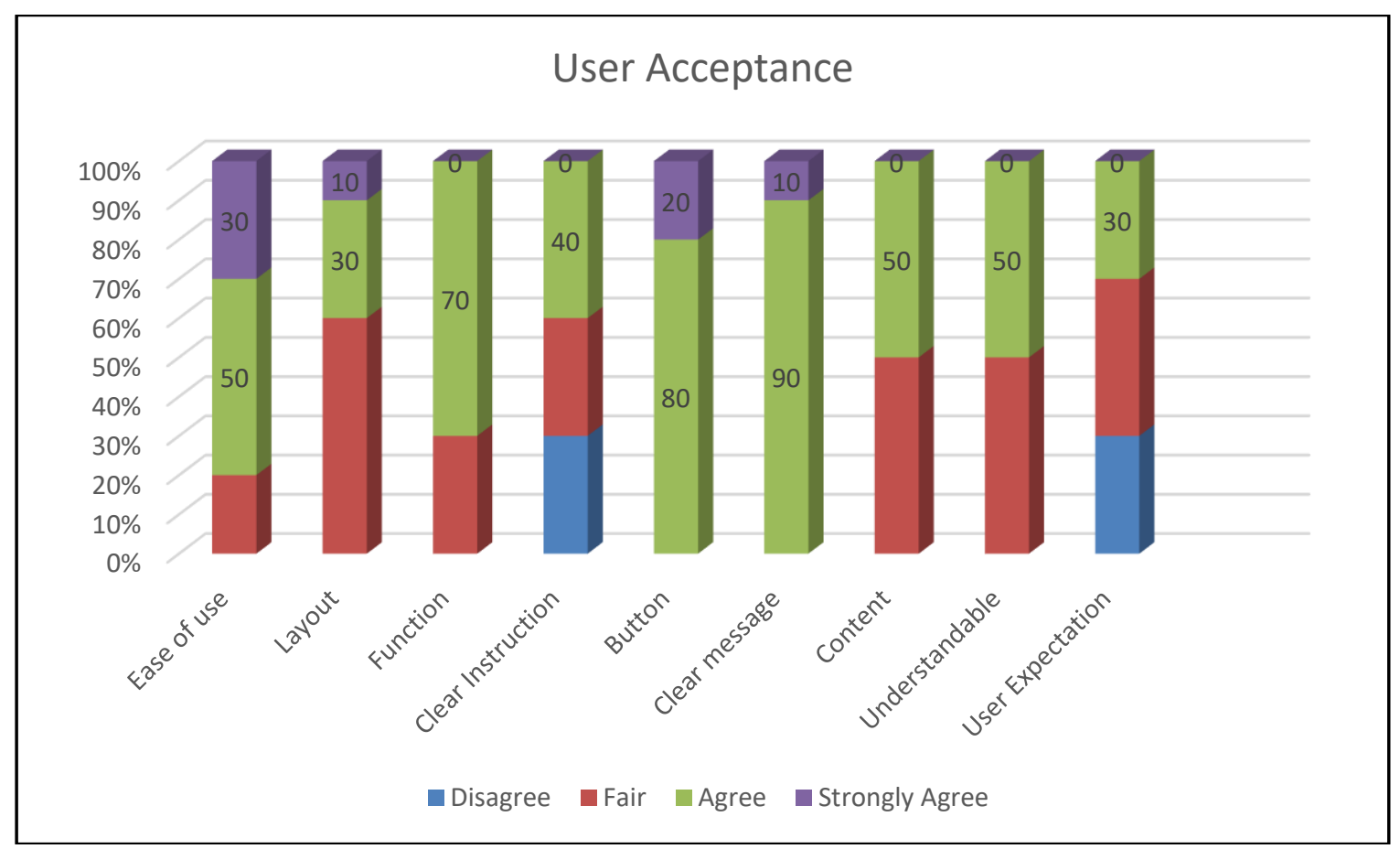

Figure 5: User Acceptance Result

Based on the result, it is shown that most of the components in the web application are well accepted by the respondents. However, there are a few components that were not achieved impressive result such as the layout, content and to understand the provided information. Thus, the interface and layout of the website should be more attractive so that can attract more people to use the website. The information also should be more meaningful and short and precise because people do not want to read too long words.

In addition, more bus company and the route can be added in the future so more people can be use the website. Besides that, the print button can be add in the future if the user wants to print details of the results in the form of hard copy. The system can be enhanced and will become useful to assist people to make a choice on the bus provider.

\section{CONCLUSION}

This paper describes the MCDM and the TOPSIS that were used in this study. Besides that, this paper also describes about the criteria that have been considered when choosing the bus transportation company. The TOPSIS method is used in this study to generate the result and give the suggestion for the best bus transportation service. It can help the users who want to use the bus transportation but do not know which bus can meet their criteria. In order to use the TOPSIS method in the system, the method was modified so that it can be implemented in PHP language. Future work will involve the control for the users who give the review for the bus transportation services. 


\section{REFERENCES}

Aruldoss, M., Lakshmi, T. M., \& Venkatesan, V. P. (2013). A Survey on Multi Criteria Decision Making Methods and Its Applications. American Journal of Information Systems, 1(1), 31-43.

Dell'Olio, L., Ibeas, A., \& Cecín, P. (2010). Modelling user perception of bus transit quality. Transport Policy, 17(6), 388-397. doi: http://dx.doi.org/10.1016/j.tranpol.2010.04.006

Eboli, L., \& Mazzulla, G. (2011). A methodology for evaluating transit service quality based on subjective and objective measures from the passenger's point of view. Transport Policy, 18(1), 172-181. doi: http://dx.doi.org/10.1016/j.tranpol.2010.07.007

Lai, W.-T., \& Chen, C.-F. (2011). Behavioral intentions of public transit passengers-The roles of service quality, perceived value, satisfaction and involvement. Transport Policy, 18(2), 318-325. doi: http://dx.doi.org/10.1016/j.tranpol.2010.09.003

Lertprapai, S. (2013). Review: Multiple Criteria Decision Making Method with Applications. Paper presented at the International Mathematical Forum.

Li, M. (2013). A multi-criteria group decision making model for knowledge management system selection based on TOPSIS with multiple distances in fuzzy environment. Kybernetes, 42(8), 1218-1234. doi: doi:10.1108/K-05-2013-0095

Purjavad, E., \& Shirouyehzad, H. (2011). A MCDM approach for prioritizing production lines: a case study. International Journal of Business and Management, 6(10), p221.

Velasquez, M., \& Hester, P. T. (2013). An Analysis of Multi-Criteria Decision Making Methods. International Journal of Operations Research Vol, 10, 56-66. 